\title{
Small scale water recycling systems - risk assessment and modelling
}

\author{
C. Diaper*, A. Dixon**, D. Butler**, A. Fewkes***, S. A.Parsons*, M.Strathern*, T.Stephenson* \\ and J. Strutt* \\ *S chool of W ater S ciences, C ranfield University, C ranfield, B edford, MK 40 OAL, UK \\ **Imperial C ollege of S cience, Technology and Medicine, London, SW 7 2BU, UK \\ ***The Notting ham Trent University, B urton S treet, Nottingham, NG 1 4BU, UK
}

\begin{abstract}
This paper aims to use quantitative risk analysis, risk modelling and simulation modelling tools to assess the performance of a proprietary single house grey water recycling system. A preliminary Hazard and O perability study (HAZOP) identified the main hazards, both health related and economic, associated with installing the recycling system in a domestic environment. The health related consequences of system failure were associated with the presence of increased concentrations of micro-organisms at the point of use, due to failure of the disinfection system and/or the pump. The risk model was used to assess the increase in the probability of infection for a particular genus of micro-organism, Salmonella spp, during disinfection failure. The increase in the number of cases of infection above a base rate rose from $0.001 \%$ during normal operation, to $4 \%$ for a recycling system with no disinfection. The simulation model was used to examine the possible effects of pump failure. The model indicated that the anaerobic COD release rate in the system storage tank increases over time and dissolved oxygen decreases during this failure mode. These conditions are likely to result in odour problems.

Keywords G reywater; in-house recycling; risk analysis; reuse simulation
\end{abstract}

\section{Introduction}

The installation of single household greywater recycling schemes has increased rapidly over the recent years and there are now a number of trial sites where systems are operating in new build developments. The interest in these systems is generated primarily by the possible $30-35 \%$ reduction in water consumption and consequent reduction in water bills. The systems are also marketed as being relatively simple and with low running costs. The basic system collects water from baths, showers and washbasins. Collection is followed by a treatment stage, usually consisting of simple filtration to remove large solids, followed by disinfection. The disinfection chemical used varies from system to system and hypochlorite, chlorine dioxide and bromine have all been utilized. Most of the systems on the market also specify that the treated product from the system can be used for flushing toilets or for garden irrigation.

At present there are no guidelines or legislation describing the installation, operating conditions or water quality requirements for water recycling systems in the UK. Risk assessment tools have been used to develop quality standards for potable water and they have been used in other countries for water reuse systems (Shuval et al., 1997). The aim of this current work is to use specific qualitative and quantitative risk analysis techniques coupled with system simulation models to assess water quality parameter guidelines for recycled water and also to link water recycling technology with building infrastructure design and policy in the urban environment (Jeffrey et al., 2000).

The testing of these water recycling systems in real situations is difficult because of the intrusive nature of monitoring and sampling. The National Water Demand Management Centre survey (Sayers, 1998) analyses efficiency and operational data and also daily 
accounts kept by the system users. Although this survey provides useful information on the operation of single household water recycling schemes the triallists were volunteers and not necessarily representative of the general population. By using risk analysis tools the system can be rigorously tested without the need for intrusive techniques and also system improvements and their effects can be considered.

\section{Methods}

The single household water recycling system was analysed using a hazard and operability study (HAZOP) performed by a multi-disciplinary team. The system was split into individual process units (Figure 1) and the causes and consequences of deviations from normal operation for each component in that unit were recorded.

A risk model of the system was formulated and used to assess microbial risks associated with the system when operating normally and during specific abnormal operating conditions identified in the HAZOP. A simulation model was also used to provide performance indicators in terms of water quality and water savings.

Risk model

The risk model combines information and data available describing: the growth of microorganisms, the removal efficiency and hydraulic characteristics of the technology under test, the epidemiology, exposure routes and thus ingestion doses and infectious doses of the micro-organisms of concern in water recycling systems (Figure 2).

Previous workers have reported that from a public health standpoint microbial contamination is the greatest cause for concern when considering reuse systems (Hrudey and Raniga, 1980; Yates and Gerba, 1998) and research has shown that there are significant quantities of faecal material in greywater (Robinson, 1996; Jefferson et al., 2000). The risk model was run for normal operation and for specific component failure for the microbial contaminant Salmonella spp. Although there is no reported evidence of Salmonella in greywater this is due to insufficient monitoring. Salmonella spp. were chosen for this study due to the availability of data and the recognised consequences of infection.

The model has been designed to accommodate data from different sources and so microbial growth kinetics are described by either the Monod or logistic equations. Monod kinetics (Equation 1) describe growth in a continuous reactor.

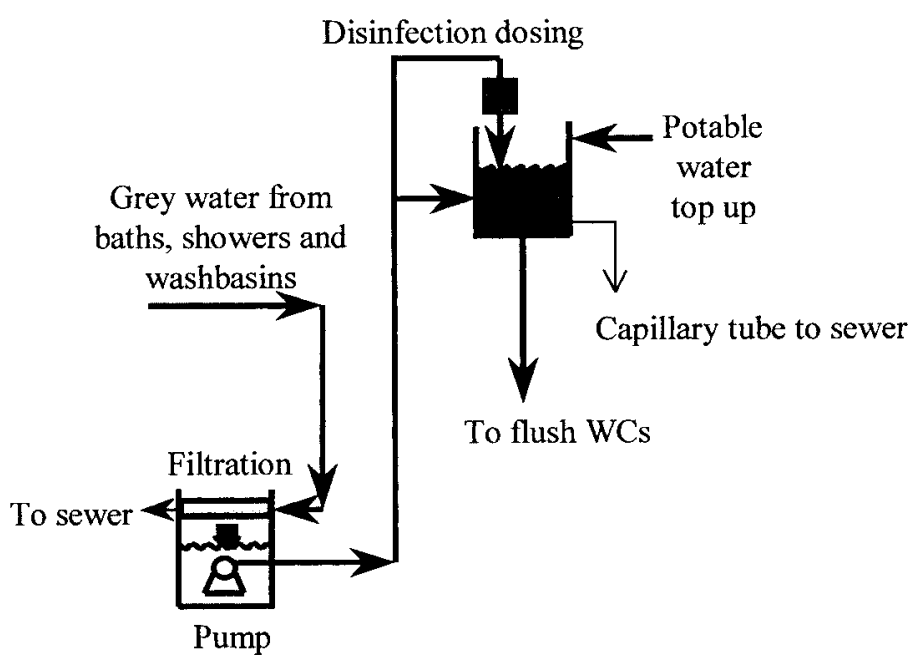




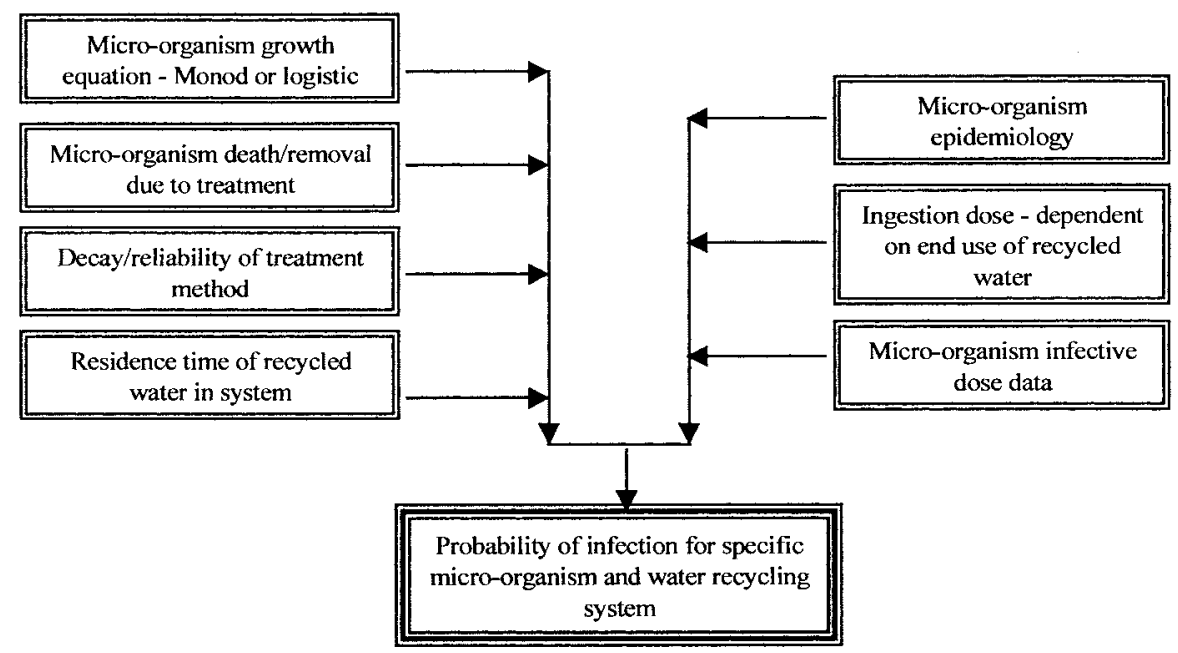

Figure 2 Schematic of the risk model

$\frac{d x}{d t}=x\left[\frac{\mu_{\max } \cdot S}{K_{s}+S}\right]$

where $x$ is the concentration and $\mu_{\max }$ is the maximum specific growth rate of the microorganism, $S$ is the concentration and $K_{S}$ is the saturation coefficient of the limiting substrate. Logistic kinetics have also been used to describe cell growth (Equation 2)

$\frac{d x}{d t}=a \cdot x\left(1-\frac{x}{x_{m}}\right)$

where $\alpha$ is the unconstrained growth rate and $x_{n}$ is the maximum sustainable niche size for the micro-organism.

Experiments with greywater have shown that aerobic microbial growth is limited by nitrogen and phospherous concentrations (Jefferson et al., submitted). However, previous workers have reported no appreciable growth or decay of Salmonella typhimurium in greywater over a period of four days (Rose et al., 1991). Thus, using an initial concentration of $10^{5}$ faecal coliforms per $100 \mathrm{ml}$ (Rose et al., 1991) and assuming the faecal coliform to Salmonella ratio is 1:1 and that all Salmonella spp. behave in a similar manner, implies a constant concentration of $10^{5}$ organisms for raw greywater.

In ideal conditions, the death rate of micro-organisms due to the treatment technology is described by either a first order death rate model (Equation 3) or a log reduction model (Equation 4).

$\frac{d x}{d t}=x \cdot k \cdot C$

where $k$ is the kill rate and $C$ is the concentration of disinfecting agent

$x=10^{\log _{10}\left(x_{o}\right)-n}$

where $x_{\mathrm{O}}$ is the micro-organism concentration before treatment and $n$ is the log reduction achieved by the technology. For the single house grey water recycling system under test, the log reduction model is used to describe the removal of a micro-organism by chlorine and is combined with a first order equation describing the rate of decay of chlorine 
concentration over time. A $\log _{10} 4$ reduction was used (Sayers, 1998) assuming Salmonella sp. behave in a similar manner to faecal coliforms in a greywater recycling system.

The equation describing the probability of ingesting a dose of micro-organism in a given time assumes a Weibull distribution. Exponential, $\beta$ Poisson or logNormal models are used for calculating the probability of infection for a specific micro-organism (Regli, 1993). For Salmonella the exponential model was used and the $\mathrm{ID}_{50}$ (infective dose) was taken as 100 organisms (Hunter, 1997).

\section{Water reuse simulation model}

The water reuse simulation model comprises of the input, routing, system and output modules (Figure 3). The input module generates or processes the characteristics of the reuse system in terms of the appliance type and system users. The Monte Carlo technique is used to generate appliance event data from frequency distributions of existing data i.e. the number and timing of baths, showers and WC flushes. The input module can also process existing time series from field study data of water usage. The routing module describes the pathways of flow within the model from raw feed stream to discharge to sewer.

The system module simulates the flow of water through each component of the reuse system. For the grey water system under investigation the system module has been calibrated to the results of laboratory characterisations. The output module monitors the system operation and generates data in the form of performance indicators describing water quality both in terms of health effects and aesthetic characteristics, water savings and cost benefits.

\section{Results and discussion}

As many of the hazards identified from the HAZOP were inter-related they are shown diagramatically in Figure 4. The hazards reported are those not covered by current regulations (UK Water Byelaws) or already corrected by the system manufacturer.

Additional consequences and causes not related to those shown above are given in Table 1. Analysis of the consequences and causes advocates the inclusion of the following recommendations in the operating manual of the system.

1. Do not clean exceptionally dirty items in washbasins or baths.

2. Specify maximum loading of sump tank.

3. System clean procedure in the event of a sewer blockage.

4. Detailed installation procedures for pump, filter, disinfection tank, sump tank and piping runs.

5. Routine maintenance of pump recommended.

6. A trouble shooting section.

In addition to maintenance and operating procedures some changes in the hardware of the system are recommended.

1. Ensure system design allows complete draining of the system when required

2. A status light on the pump to ensure system users are aware of pump failure

3. An "over pressure" or "no flow when operating" cut out on the pump

4. A pump low flow alarm or indicator light

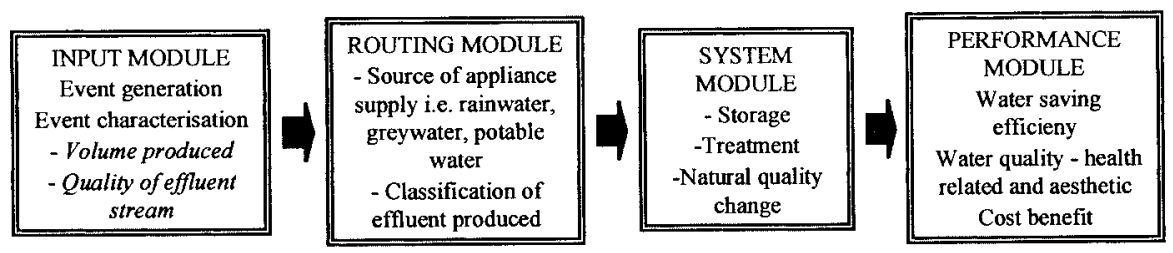

Figure 3 Schematic of the reuse simulation model 


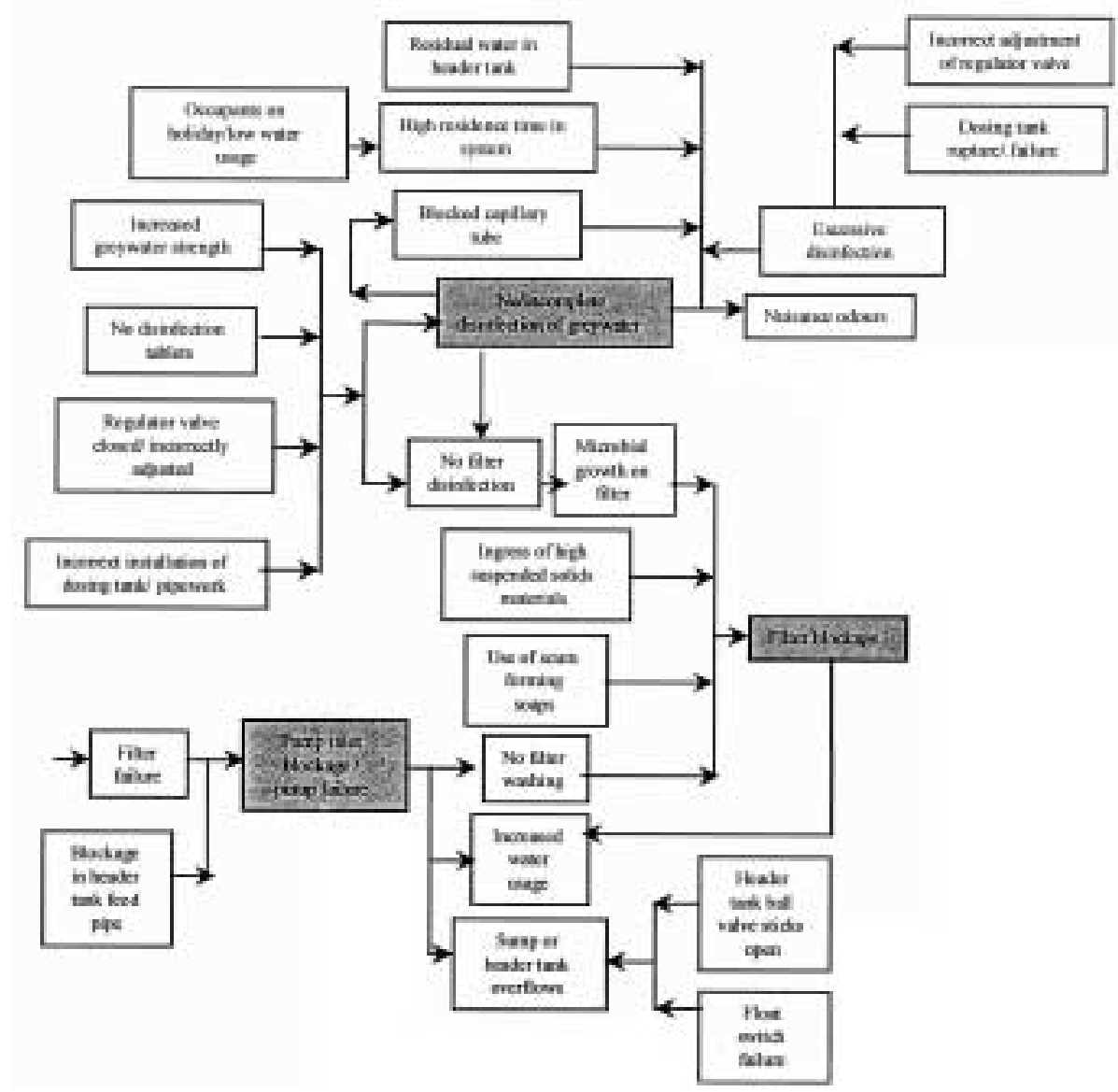

Figure 4 Schematic of HAZO P consequences/causes resulting from deviations in operation of single house greywater recycling system

Table 1 Additional consequences/causes resulting from deviations in system operation

\begin{tabular}{ll}
\hline Consequences & Cause \\
\hline Higher/lower residence times in system & Normal operation \\
B locked capillary tube & Microbial growth/ solids build up \\
Sump tank failure & Excessive load \\
Ingress of sewage into sump and header tanks & S ewer blockage \\
Release of halogens & Use of certain domestic cleaning products \\
No water to flush toilets & Ball valve stuck off \\
No drainage of water from baths/sinks & Blockage in collection pipework \\
\hline
\end{tabular}

A number of the causes and consequences required further investigation and work is currently being undertaken to identify domestic household cleaning materials that could result in the release of halogens or that could cause corrosion to the filter. Also, reliability data for pumps, valves and level switches is required in order to calculate the possible frequency of particular hazardous events.

System failures that could lead to increased water usage, the production of odours or increased microbial growth were investigated further using the risk and simulation models. Scenarios selected were: no disinfection, filter blockage and pump failure. 
Risk model

The probability of contracting a salmonella infection given the presence of Salmonella spp. in the initial greywater was calculated to be less than $1.5 \times 10^{-7}$ if the disinfection system is operating correctly and less than $1.5 \times 10^{-3}$ if there is no disinfection. In the general population number of reported cases of salmonella per year is 30,000 (PHLS website). However, a degree of under reporting occurs and this is assumed to be $50 \%$, thus a rate of 60,000 cases is used. Other assumptions include: an infected person sheds organisms into the greywater system for 2 days, the greywater system is flushed through completely within a day and on average 4.4 people would be exposed to the system in any day.

Initial calculations estimate an increase of $<0.001 \%$ in the number of cases of Salmonella spp. infection for householders using a greywater system with a fully working disinfection system. For a greywater system in which there is no disinfection an increase of approximately $4 \%$ is calculated.

\section{Water reuse simulation model}

The water reuse simulation model was used to indicate the effect of failure of the pump on the dissolved oxygen (DO) concentration (Figure 5), anaerobic COD release rate (Figure 6). These parameters were selected as DO concentration and anaerobic COD release rate are linked to the production of odours and the DO concentration will relate to the growth of aerobic micro-organisms. Data was generated for the quality parameters for a range of temperatures, as temperature was found to significantly effect the curves generated.

The DO concentration was shown to decrease over time as expected for a stagnant tank. This can be linked with the anaerobic COD release rate, which was shown to increase more rapidly at higher temperatures where the DO decreases rapidly. This suggests that at higher temperatures the production of "off" odours is more likely.

A final scenario simulated by the model was the effect of gradual filter blockage on the water saving efficiency of the system. The HAZOP suggested that filter blockage could be caused by a number of situations and would lead to an increased water usage (Figure 4).

The effect can be shown in terms of cleaning frequency, the more often the filter is cleaned the less the degree of blockage of the filter (Figure 7). For the specific conditions stated for this simulation a cleaning frequency of $<4$ weeks is recommended.

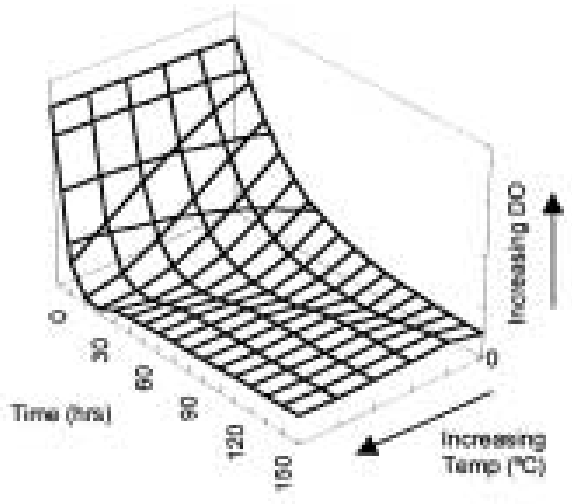

Figure 5 Effect of pump failure on dissolved oxygen concentration in header tank for a range of temperatures

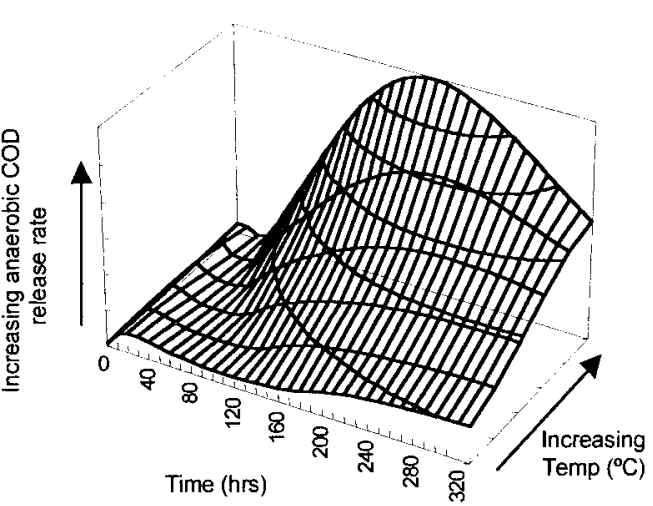

Figure 6 Effect of pump failure on anaerobic C O D release rate in header tank for a range of temperatures 


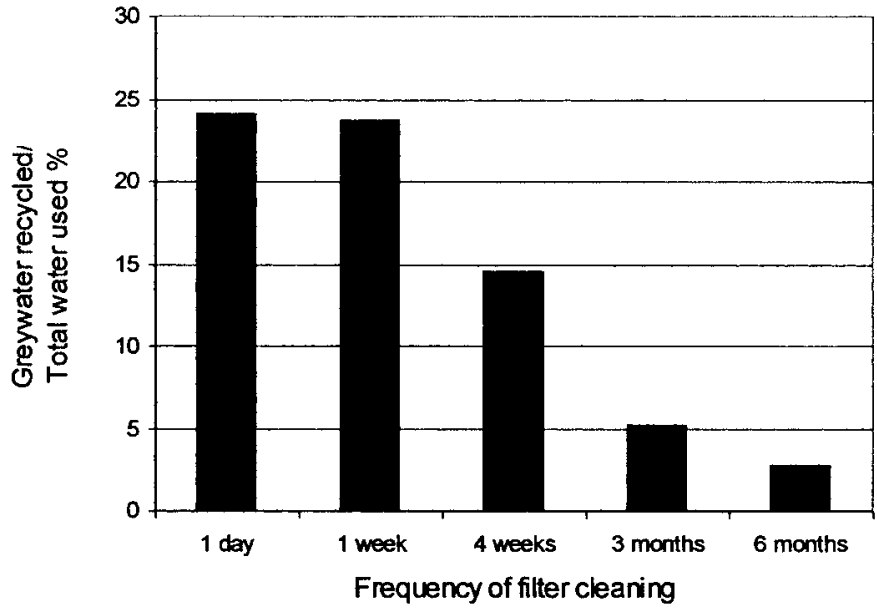

Figure 7 Effect of filter cleaning frequency on \% greywater recycled

\section{Conclusions}

A number of operational and installation requirements should be included in the manual of single house greywater recycling systems to ensure the health and safety of the users and the correct operation of the system. Fundamental requirements should include the installation of key items of equipment and maintenance procedures for the pump. A system cleaning procedure and trouble shooting guide should also be included and the recommendation that exceptionally dirty items should not be cleaned in the bath or hand washbasin. Improvements to the hardware of the system are required, mainly relating to the pump, a critical item of equipment.

The reliability of the disinfection system is also vital in view of the calculated increase from $0.001 \%$ to $4 \%$ in the number of cases of infection for a specific micro-organism. There is a paucity of information on the growth kinetics and epidemiolgy of different microorganisms in grey water and a number of assumptions were made in this calculation. Therefore, in order to verify this calculation for the number of cases of infection, the risk model will be used with set parameter ranges to estimate the error in these values. Future work will also use event tree analysis to estimate the possible frequency of disinfection system failure.

The water reuse simulation model provides a useful tool in assessing the consequences of different system failure modes. Temperature was shown to be an important factor in modelling the water quality during a pump failure scenario. As the current research programme includes the monitoring of real greywater systems, future work will involve full calibration of the model, which will improve the accuracy of simulations. Also, once fully calibrated the simulation model will be integrated with the risk model, as output data on micro-organism growth from the simulation model will provide input data to the risk model.

\section{References}

Hrudey, S.E and Raniga, S. (1980). Greywater Characteristics Health Concerns and Treatment Technology,

Design of Water and Wastewater Service for Cold Climate Communities. Proceedings of Post

Conference Seminar in Conjunction with IAWPR Conference, Toronto, 137-154.

Hunter, P.R (1997). Waterborne Disease: Epidemiology and Ecology. Wiley, Chichester.

Jefferson, B., Laine, A., Judd, S.J. and Stephenson, T. (2000). Membrane bioreactors and their role in waste- 
Jeffrey, P., Parsons, S.A., Judd, S.J., Stephenson, T., Seaton, R.A.F., Fewkes, A., Butler, D. and Dixon, A. (2000). An interdisciplinary approach to the assessment of water recycling technology options. Urban Water (in press).

Jefferson, B., Burgess, J.E., Pichon, A., Quarmby, J. and Judd, S.J., (Submitted). Nutrient supplementation to enhance the biological treatment of greywater. Wat. Res.

PHLS website, http://www.phls.co.uk/facts/salm-t01.htm

Regli, S. (1991). Modelling the risk from giardia and viruses in drinking water. JAWWA, 83(11), 76-84.

Robinson, M. (1996). The storage and recycling of domestic greywater. Unpublished MSc/DIC dissertation, Imperial College of Science, Technology and Medicine, 1996.

Rose, J.B., Gwo-Shing Sun, Gerba, C.P. and Sinclair, N.A. (1991). Microbial quality and persistence of enteric pathogens in greywater from various household sources. Wat. Res. 25(1), 37-42.

Sayers D,. (1998). A study of domestic greywater recycling. National Water Demand Management Centre, Environment Agency, Worthing.

Shuval, H., Lampert, Y. and Fattal, B., (1997). Development of a risk assessment approach for evaluation wastewater reuse standards for agriculture. Wat. Sci. Tech 35(11-12), 15-20

Yates, M.V. and Gerba, C.P. (1998). Microbial considerations in wastewater reclamation and reuse. In: Wastewater Reclamation and Reuse, Asano T (ed.), 1st edn, Technomic Publishing Co, Lancaster, USA pp.437-488. 